\title{
Bipolar Affective Disorders and Psychotic Disorders in War Torn Area Eastern of the Democratic Republic of Congo
}

Mutume Vivalya ( $\sim$ nzanzumutume@kiu.ac.ug )

Kampala International University - Western Campus https://orcid.org/0000-0003-2068-2830

Martial Mumbere Vagheni

Universite de Kinshasa

Kalima Nzanzu Adelard

Catholic University of Graben

Kasereka Masuka Rock

Catholic University of Graben

Manzekele Bin Kitoko

Universite de Kinshasa

Wilson Mugizi

Kampala International University - Western Campus

Scholastic Ashaba

Mbarara University of Science and Technology

Research article

Keywords: Bipolar, Prevalence, Psychotic, Disorders, War, Traumatic.

Posted Date: June 3rd, 2020

DOl: https://doi.org/10.21203/rs.3.rs-21746/v2

License: (c) (i) This work is licensed under a Creative Commons Attribution 4.0 International License.

Read Full License 
The authors have withdrawn this preprint from Research Square 